\title{
Separation of locally determined work piece deviations and measurement uncertainties for structured-light scanning of customized polymer gear wheels
}

\author{
Andreas Michael Müller ${ }^{1}$, Sebastian Metzner ${ }^{1}$, Tino Hausotte ${ }^{1}$, Dominik Schubert ${ }^{2}$, Dietmar Drummer ${ }^{2}$ \\ ${ }^{1}$ Institute of Manufacturing Metrology (FMT), Naegelsbachstrasse 25, 91052 Erlangen, Germany \\ ${ }^{2}$ Institute of Polymer Technology (LKT), Am Weichselgarten 9, 91058 Erlangen-Tennenlohe, Germany
}

\begin{abstract}
Summary
During the manufacturing of work pieces, geometrical deviations from the intended nominal geometry of the designer are inevitable. The procedure of conformance testing defined in ISO 14253-1:2018-07 is used to ensure the function of a work piece by verifying the geometrical compliance with pre-defined tolerance specifications. Depending on the measurement setup used for the validation step and the accuracy of the manufacturing process, it is possible that the measurement uncertainty is large enough to have a significant influence on the conformance evaluation. The measurement uncertainty for optical measurement systems is influenced by the surface properties of the test specimen. This contribution aims to demonstrate the complete workflow for the determination of the single point uncertainties for a given measurement task in order to separate the local work piece deviations from the systematic and random components of the measurement uncertainty. It could be shown for a demonstration scenario that different necessary coloring methods of polymer gear wheels, which are required to enable measurements using structured-light scanning, have a measureable influence on the local distribution of the measurement uncertainty. This information could then be used for downstream processes in various use cases, e.g. for the improvement of holistic tolerance simulation models or the improvement of geometrical measurements using weighted regression analysis.
\end{abstract}

Keywords: structured-light scanning, work piece deviations, measurement uncertainty, gear wheel, colored polymer

\section{The concept of the single point uncertainty}

Generally, the results of any geometrical measurement of a work piece and the subsequent comparison against the nominal geometry (CAD model) as defined by the designer can be divided into three different contributions: a) the work piece deviations with respect to the nominal geometry, b) the (signed) systematic measurement error of the measurement system and c) the (unsigned) random measurement error of the measurement system. The measurement uncertainty is a positive non-zero parameter associated with every real measurement and can only be reduced but never be completely avoided. The "Golden rule" of metrology states that the measurement uncertainty shall be less than $10 \%$ to $20 \%$ of the tolerance [1-3]. This requires a close observation of the achieved measurement uncertainties for each geometric verification task. Recent research efforts at the Institute of Manufacturing Metrology (FMT) dealt with the question of how to determine the local distribution of the measurement uncertain- ties over the surface of a work piece and subsequently derive metrological benefits from that knowledge. The developed framework of the single point uncertainty (SPU) requires several inputs:

- A measurement object.

- The nominal geometry of the measurement object (CAD model).

- A measurement system targeted for the uncertainty examination and capable of recording complete areal measurements of the chosen measurement object.

- A statistically significant number of measurement repetitions (here 20 , following guideline VDI/VDE 2630 [4] and ISO 15530-3:2011 [5]).

- A single reference measurement of the measurement object (reference geometry) performed by a reference measurement system in the sense of VIM [6] which is also capable of recording areal measurements of the chosen measurement object. 
- A suitable sampling strategy dependent on the processed geometries and the computational resources available.

At first, a reference measurement of the measurement object needs to be created using the reference measurement system. The captured geometry (usually represented by a triangle mesh in the STL format) is then geometrically aligned against the nominal geometry (CAD) by means of a geometrical registration routine. One property of a reference measurement system is that the measurement uncertainties for that specific measurement task is known to be low compared to the expected geometrical deviations. Consequently, the observed geometrical differences between the nominal geometry and the reference measurement can be fully assigned to the work piece deviations. Then, a measurement series consisting of $n$ repeated measurements of the same measurement object is created, using the measurement system chosen for the uncertainty evaluation. Each measurement is then geometrically registered against the reference measurement. After the alignment of the surfaces, the calculation of the spatial measurement uncertainty distribution can be performed using different sampling strategies (Fig. 1). If the normal vectors of the reference geometry are trustworthy and the expected measurement uncertainty is rather small compared to geometric features on the surface, the sampling strategy "normal vector" can be used (Fig. 1, right). Starting from each sampling point $S P_{k}$ on the reference geometry, the intersection distance of a constructed search ray in the direction of the vertex normal vector is calculated for each measurement repetition. Thus, the resulting sets of distances $\left(d^{k}=d_{i}^{k}=\right.$ $\left.\left\{d_{1}^{k}, d_{2}^{k}, \ldots, d_{n}^{k}\right\}\right)$ are effectively assigned to their respective nominal surface vertices $S P_{k}$, which effectively maps the computed distances onto the nominal geometry.

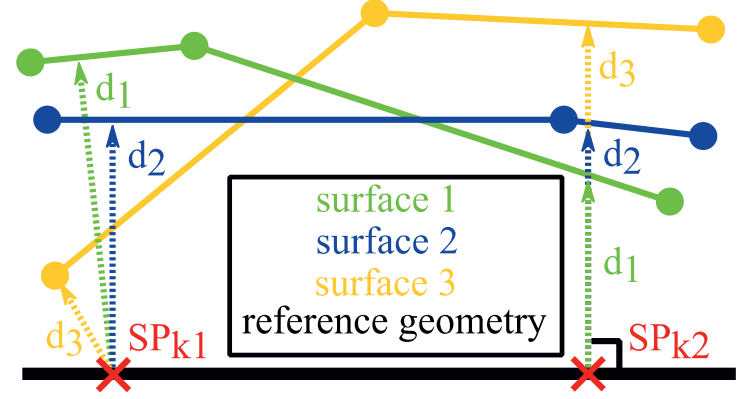

Fig. 1: Visualization of different sampling strategies: "shortest distance" (left) and "normal vector" (right).

The detected distances $d^{k}$ per definition only represent contributions to the measurement un- certainty of the used measurement system, because the reference geometry by agreement represents the true geometry of the work piece. Consequently, the mean value of $d^{k}$ represents the local systematic measurement error, while the local distribution of random measurement errors (precision) is determined by the standard deviation of $d^{k}$. After that, a suitable sampling method is applied to determine the local work piece deviations for each sampling point $S P_{k}$ by comparing of the (same) reference surface against the nominal geometry. Summarizing the above, the single point uncertainty (SPU) is a framework to determine the geometric deviations and both components of the associated measurement uncertainty for each sampling point $S P_{k}$ on the surface of a reference geometry, thus drawing a spatially resolved uncertainty map of a specific measurement task. It is advantageous if the reference surface is pre-processed in such a way that the triangles of the mesh have uniform properties, thus resulting in a homogeneous and preferably very dense sampling point distribution. Compared to the sampling strategy "normal vector", the method "shortest distance" can result in better results in edge regions or if large distances are expected to be computed (Fig. 1). Nonetheless, in general both methods result in very similar uncertainty characteristics with the main difference being the computation requirements and the easiness with which certain run time sensitive optimization measures can be implemented [7]. In case no reference measurement is available, the method can be used nonetheless to compute the areal distribution of the random measurement error, while the work piece deviations and the systematic measurement error cannot be separated this way. It is important to note, that the determination of the SPU can be rather sensitive to geometrical misalignments resulting from a poor registration routine, which requires a certain diligence when deciding for a registration strategy. Extensive research at the Institute of Manufacturing Metrology (FMT) has revealed numerous different usage scenarios, in which the determination of the SPU can better the understanding of a measurement setup and consequently improve the measurement itself. It was successfully shown in [8] that locally varying systematic measurement errors of a real industrial X-ray computed tomography (CT) system could be corrected by the determination of the SPU of repeated simulated measurements, if the simulation framework is sufficiently adopted to the real CT system. The information about the local measurement precision was successfully used within the implementation of a weighted geometry element regression analysis (also called fit / fitting), resulting in improved 
measurement results [9]. Fits are commonly used to assign standard geometry elements to measurement coordinates by minimizing a certain error function in order to determine the size and orientation of a specific geometry element (e.g. fitting of a cylinder to the point cloud representing a measured drilling). The method also allows the transfer of information about the measurement uncertainty associated with a certain measurement task to the downstream process of tolerance management [10]. Here, the measurement uncertainty can then be taken into account during the design of work pieces and determination of the associated tolerances. The determination and evaluation of the SPU yields the best results for measurement systems, which produce an areal representation of the work piece geometry (e.g. computed tomography, structured-light scanning in combination with a rotatory axis and focus variation). Nonetheless, similar evaluations are also possible for tactile coordinate measurement machines, although a different data processing pipeline needs to be implemented and utilized [11].

\section{The gear wheel measurement object}

The gears examined in this contribution (Fig. 2, $\varnothing$ approx. $39 \mathrm{~mm}$ ) were produced by injection molding using a Polyoxymethylen (POM) of the type Hostaform C9021 by Celanese Services Germany $\mathrm{GmbH}$, Kelsterbach, Germany. POM is often used in gear applications due to its selflubricating properties and resulting good dry running capabilities and low friction and wear [12]. The semi-crystalline structure of the material leads to a process dependent microstructure. In injection molding processes, an optically amorphous edge layer is formed due to the high cooling rate in the areas with contact to the mold material. This leads to the formation of crystalline nanostructures with size below the wavelength of visible light. As a result, the edge layer appears optically amorphous [13]. This semitransparent edge layer leads to difficulties in optical measurements, especially with stripe light projection methods, due to the scattering of the projected lines into the material. Thus, the surface cannot clearly be determined. A further factor complicating optical measurements on this material is its gloss. To achieve useful measurements, the optical properties of the material have to be altered.

Using colorants i.e. pigments with or without carrier materials, the optical properties of polymer materials can be altered easily and cost-efficiently. Pigments and other nano-sized particles act as scattering centers, reducing the translucency of the optically amorphous skin layers. They can also decrease the amount of directed reflection, reducing gloss [14]. There are three main methods of colorizing polymers with pigments. Firstly, pigments can be added to the base material to produce pre-colored granulate. This is often used for large batches of material and frequently used colors such as black and white. Secondly, master batches, highly pigment-filled granules, are used to color smaller batches individually. Thirdly, liquid colors can be used instead of master batches, allowing for a more flexible mixing of pigments and a wider range of colors. The cost of coloration depends on choice of pigment, way of coloration and dosage. Master batches and liquid colors are more expensive than pre-dried material, but they are more flexible. Pigment cost can vary widely from inexpensive carbon to more expensive mineral or metal based effect pigments. Due to the complex interaction of the pigment particles, carrier materials and polymer materials, there is no single generalized effect of colorants on the resulting properties of polymers. However, most pigments act as a nucleating agent, increasing the degree of crystallinity, leading to higher shrinkage and therefore smaller parts [15].

To exclude influences of carrier materials, two pigment-based colorants were chosen for this research. The pigments were added by means of direct compounding in order to achieve a nontranslucent, low-gloss surface with homogenous pigment distribution to improve the optical properties of the gear specimens for further optical measurements. The following two pigments were used to color the POM gear wheels (Fig. 2):

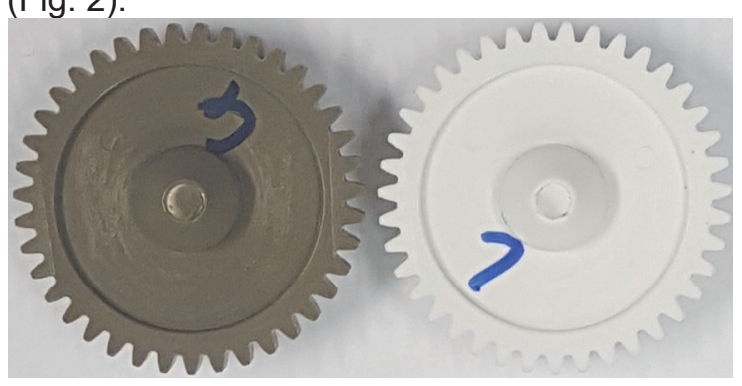

Fig. 2: Colored gear wheels: IDDG (left) and IDw (right)

i. A pigment mixture in the ratio of 1:1 of a yellow chrome antimony titaniumoxide rutile pigment ((Ti,Cr, Sb) O2) of the type Sicotan Gelb K 2001 FG, supplied by BASF Pigment $\mathrm{GmbH}$, Besigheim, Germany, with a Carbon Black (C) pigment of the type Dei®Pow - Spezialschwarz, also provided by Deifel $\mathrm{GmbH} \& \mathrm{Co}$. KG. The identifier (ID) for the corresponding measurement object is $I D_{\mathrm{DG}}$ (DG: dark green, Fig. 2, left).

ii. A white titanium dioxide (TiO2) pigment of the type DeißPow - Weiß, provided by Deifel $\mathrm{GmbH}$ \& Co. KG, Schweinfurt, Germany. The 
identifier (ID) for the corresponding measurement object is $I D_{\mathrm{w}}(\mathrm{W}$ : white, Fig. 2, right).

An injection molding machine of the type Arburg 370 U-700-30-30 with a plasticizing unit with $18 \mathrm{~mm}$ screw diameter by Arburg $\mathrm{GmbH}$ \& Co. KG, Loßburg, Germany, was used to produce the gear specimens. All gear wheels were produced using the same tool. Table 1 shows the processing parameters used for manufacturing.

Table 1: Overview of processing parameters

\begin{tabular}{|l|l|l|}
\hline Parameter & Unit & Value \\
\hline Mass temperature & ${ }^{\circ} \mathrm{C}$ & 205 \\
\hline Mold temperature & ${ }^{\circ} \mathrm{C}$ & 105 \\
\hline Injection speed & $\mathrm{mm} / \mathrm{s}$ & 15 \\
\hline Injection time & $\mathrm{S}$ & 1.5 \\
\hline Holding pressure & bar & 1200 \\
\hline Holding time & $\mathrm{s}$ & 28.5 \\
\hline
\end{tabular}

To ensure a correct alignment of the measured gear wheel geometries, a drilling $\varnothing 1.5 \mathrm{~mm}$ was arranged in one of the tooth roots (arbitrarily chosen) of each gear wheel before the measurements. This marker is then also captured by the measurement system and makes the correct alignment of the same teeth of different measurements possible during a manual registration step.

\section{Measurement setup and data processing}

Goal of this contribution was to evaluate the locally distributed single point uncertainties for gear wheel measurements using a structuredlight scanner. The used system was GOM ATOS CORE 200 5M [16] in combination with a rotatory stage (Fig. 3 ).

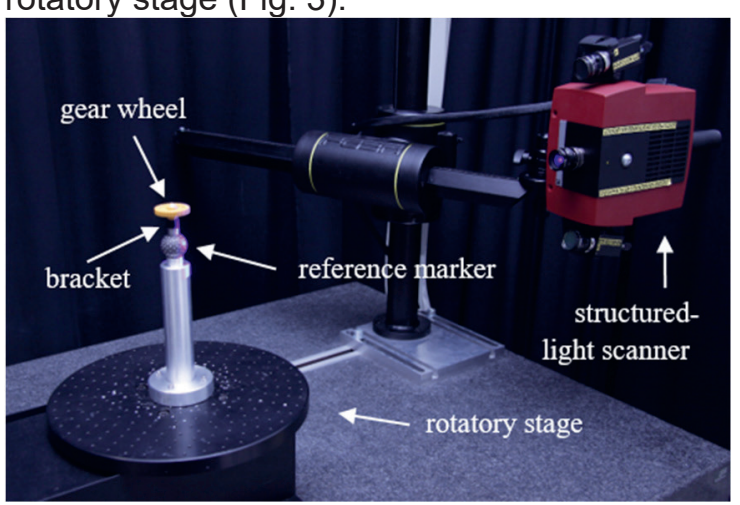

Fig. 3: Figurative representation of the measurement setup, consisting of a structured-light scanner (here GOM ATOS Compact Scan 2M / 300) in combination with a rotatory stage.

The sensor was controlled by the software GOM ATOS Professional 2018 [17]. The internal settings for the sensor were set to the least restrictive options possible, thus making sure that as many surface points as possible were recorded during the measurement. The illumination settings were determined by user-controlled selection of a target of interest on the live image once before each measurement series. During the measurements, all external light sources in the laboratory were dimmed as much as possible in order to ensure the exclusive illumination by the measuring device. The measurement object (gear wheel) was mounted onto the rotatory stage through its accessible rotatory axis. The used bracket was equipped with reference markers, which enabled the software GOM ATOS Professional 2018 to perform the referencing of each image with the angular position of the rotatory stage. The sensor is characterized by the following key data: measuring range $200 \times 150 \mathrm{~mm}^{2}$, lateral resolution $80 \mu \mathrm{m}$, measuring distance $250 \mathrm{~mm}$ and native camera resolution 5 million pixels. Before measurement, the sensor was calibrated using the calibration plate CP40 17040760 consisting of 3657 calibration points. A single measurement consisted of 200 single images (projections), which were equally distributed over a full rotation $\left(360^{\circ}\right)$ of the rotatory stage, with two different sensor positions each: One position captured the gear wheel sloping from above the gear wheel and the other from below with both angles roughly equaling $45^{\circ}$. As soon as all images were recorded from that position, the scanner was manually moved to the second position and the second set of 200 projections was recorded. The reference markers were visible to the scanner from both senor positions, which made the data fusion of several image stacks possible. Thus, the GOM software merged all of the 400 images together into one surface representation of the gear wheel in a triangulated mesh format (STL). The option "post processing" during the polygonization routine was disabled and thus all explicit filter operations disabled in order to achieve a preferably unaltered measurement result. Each measurement was repeated 20 times to ensure a statistically valid result for the following single point uncertainty calculations. The measurements were performed in a temperature-controlled environment with temperatures set to $20^{\circ} \mathrm{C} \pm 1 \mathrm{~K}$. Overall, two different gear wheels were examined, and thus 40 single measurements (each with two positions) were performed.

The reference measurements were captured using the industrial X-ray computed tomography system Zeiss METROTOM 1500 [18]. The gear wheel was positioned on the rotatory stage using the bracket already presented in Fig. 3. The upper part of the bracket consisted of polymer, which was easy to penetrate by X-rays and did not lead to CT-specific sources of errors due to 
a possibly high material density. CT systems are in general very well suited for measuring polymer parts with high accuracy. To ensure a high quality measurement, time sensitive device settings were chosen in order to guarantee a very low measurement uncertainty of the reference measurement system. Because of the fact that the polymer coloration had no measureable impact on the absorption properties of the work piece, these following settings were used for all reference measurements: tube voltage $150 \mathrm{kV}$, tube current $400 \mu \mathrm{A}$, resulting nominal focal spot size $60 \mu \mathrm{m}$, X-ray filter $0.5 \mathrm{~mm} \mathrm{Cu}$, geometrical magnification $9.0 \mathrm{x}$, resulting voxel size $22.3 \mu \mathrm{m}$, integration time $1000 \mathrm{~ms}$, number of projection averaged 10, number of projections per measurement 2050 . The required surface determination was performed using VGStudio Max 3.2 [19] using the settings: automatic grey value threshold, search distance 16 voxels, remove all particles and voids, iterative surface determination. The measured surface could then be exported as a triangle mesh in the STL format using high quality settings. The measurement was subsequently registered against the nominal geometry (CAD) using VGStudio Max. Finally, the gear sprocket was extracted from the full surface using an own MATLAB program by removing all triangles with an edge point distance to the rotatory axis of smaller than $16 \mathrm{~mm}$. The authors are aware of the fact, that an industrial CT system might not necessarily show improved measurement uncertainty characteristics compared to a structured-light scanner. Nonetheless, these measurement devices were chosen to demonstrate the principle approach for the determination and evaluation of the single point uncertainty framework as a tool in dimensional metrology.

As mentioned above, the sampling strategy "normal vector" requires trustworthy sampling vectors in order to compute the metrologically correct distances. CT measurements are often subject to high frequency noise with location wavelengths considerably higher than the structural resolution of the measurement system. Two important factors, which influence the structural resolution in a relevant way, are the geometrical magnification, which results in a certain voxel size, and the X-ray focal spot size. The structural resolution can reach sub-voxel values, and the actual value depends heavily on the measurement itself. Thus, in order to remove very high spatial frequencies and to improve the triangle mesh properties regarding triangle edge size and homogeneity, the generated surface from the reference measurement was undertaken a post-processing routine using GOM ATOS Professional 2018. First, the mesh was smoothed using the operation "Mesh $\rightarrow$
Smooth..." with settings "very large filter radius", "very low detail sharpness" and "surface tolerance $10 \mu \mathrm{m}$ ". After that, the homogeneity of the surface point distribution was improved using the operation "Mesh $\rightarrow$ other $\rightarrow$ Regularize..." with settings "surface tolerance $10 \mu \mathrm{m}$ and "maximum edge length $50 \mu \mathrm{m}$ ". Of course, these kinds of operations need to be used with special care, because any filtration of the reference measurement results in altered uncertainty values for the examined structured-light measurement device. Because of that, the effect of the described mesh operations was investigated further by comparing the filtered mesh against the unfiltered mesh using a conventional nominal-actual comparison (VGStudio Max). Figure 4 shows, that the deviations between the filtered and the unfiltered geometry nearly form a perfectly shaped Gaussian distribution with the expected value $\mu=0$. The comparison against the regression result of the deviations using a Gaussian model confirms the underlying normal distribution. Consequently, we can state, following the central limit theorem [20], that the removed part of the geometry consists of noise, thus it is part of the random measurement errors. Because of that, and because of the fact that the removed parts of the geometry are clearly below the voxel size $(22.3 \mu \mathrm{m})$ and the X-ray focal spot $(60 \mu \mathrm{m})$ of the CT system, it is stated that no relevant measurement information of the geometry form was removed by the mesh post-processing operations.

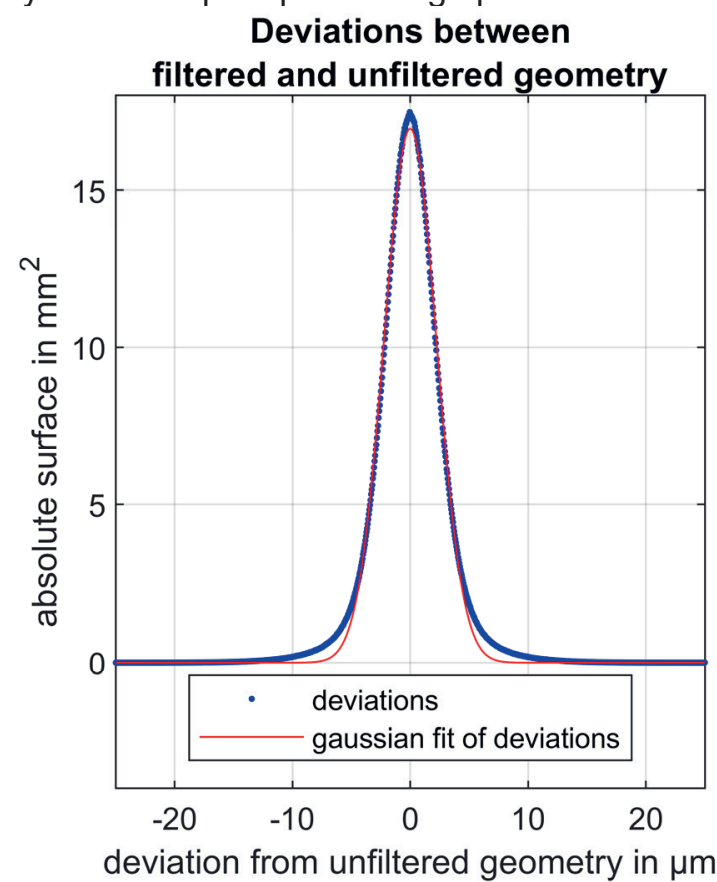

Fig. 4: Examination of the geometry altering mesh operations using a nominal-actual comparison and a Gaussian fit. 
After the post-processing of the reference geometry, each of the 20 measurement repetitions was registered against that reference measurement using VGStudio Max. Following a first rough alignment, the rotatory symmetry was dissolved by arrangement of the drilling in one of the tooth roots. Then the gear wheel sprocket was extracted by removing all triangles with an edge point distance to the rotatory axis of smaller than $16 \mathrm{~mm}$ (same procedure as for the reference measurement before). The geometrical registration was finalized by a fine registration using the settings "quality level 50", "consider current transformation", "improved refinement" and "consider surface orientation". Figure 5 shows the alignment of each single measurement with the reference geometry. It is clearly observable, that the drillings in each mesh lie on top of each other, thus confirming a valid registration result.

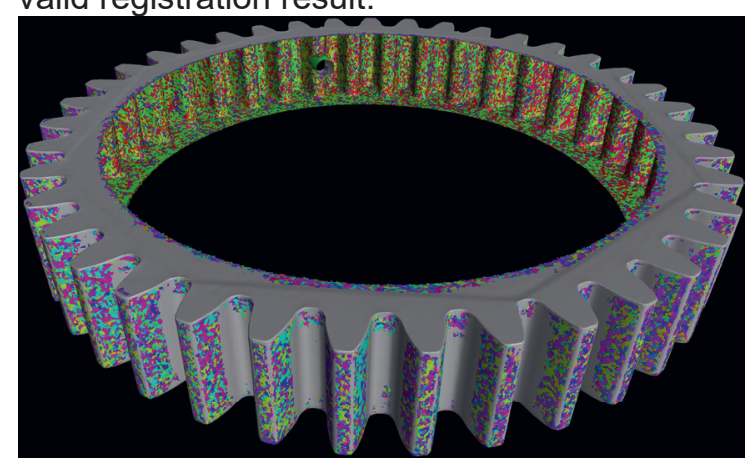

Fig. 5: Alignment of the sprocket of the reference geometry (gray) and the 20 measurement repetitions (various colors) after the registration routine.

After that, the work piece deviations were calculated by determining the distances from each sampling point of the reference geometry to the nominal geometry using the sampling strategy "shortest distance". Then, the single point uncertainty was calculated by determination of the distances from each of the same sampling points to each of the measurements of the measurement series using the sampling strategy "normal vector" (as described in the first chapter). Finally, each sampling point $S P_{k}$ was associated with a value describing the local work piece deviations $t$ and a vector $d^{k}$ characterizing the local measurement uncertainty.

\section{Results}

First, the geometrical work piece deviations were examined by calculating the local distances between the reference measurement and the nominal geometry (CAD). Fig. 6 shows that the gear exhibits some deviations at the tooth root as well as the tooth tip of around $200 \mu \mathrm{m}$, while the tooth flanks only show very small geometrical deviations compared to the nominal geometry. The systematic work piece deviations for the other gear wheel (IDw) only deviated marginally from the shown results in Fig. 6, which means that the different colored compounds were not affected differently by the used manufacturing process parameters (see also Table 1).

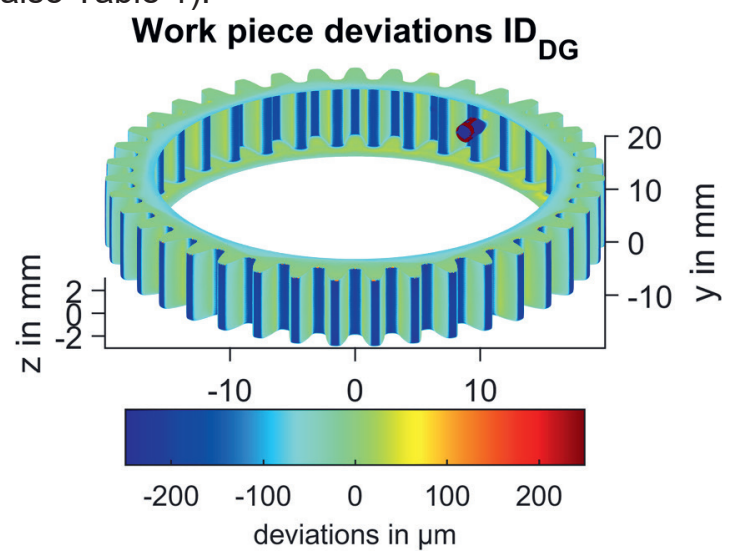

Fig. 6: Visualization of the systematic work piece deviations of a gear wheel

Figure 7 shows the expanded measurement uncertainty with a coverage factor of $k=2$ for the gear wheel measurement using a structuredlight scanner. The expanded uncertainty is calculated by the quadratic sum of the systematic and random measurement errors and is then multiplied with a coverage factor $k$. A coverage factor of 2 results in having a level of confidence of approx. $95 \%$, assuming a normal distribution [21]. The results in Fig. 7 show that the limited optical accessibility of the gear roots resulted in a substantial increase of the expanded measurement uncertainty over $100 \mu \mathrm{m}$.

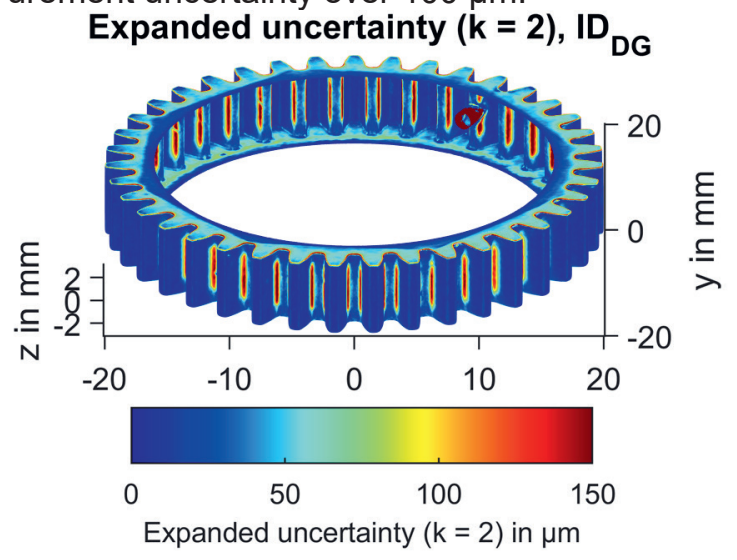

Fig. 7: Visualization of the expanded measurement uncertainty $(k=2)$ of gear wheel $I D_{\mathrm{DG}}$

The gear wheel side also exhibits a larger measurement uncertainty because of the angled image capturing. The upper part of the gear flanks and the tooth tips were measured with a 
comparably low expanded measurement uncertainty significantly below $50 \mu \mathrm{m}$. Table 2 presents a statistical view of the calculated deviations, which shows that the precision of the measurement setup was very high (P50 approx. $3 \mu \mathrm{m})$.

Table 2: Statistical view of the calculated work piece deviations and measurement uncertainty contributions (ID $\mathrm{DG}$ )

\begin{tabular}{|c|c|c|c|c|c|c|}
\hline \multicolumn{7}{|c|}{ sys. work piece deviations percentiles in $\mu \mathrm{m}$} \\
\hline P01 & P05 & P25 & P50 & P75 & P95 & P99 \\
\hline-206 & -193 & -72 & -23 & -3 & 19 & 32 \\
\hline \multicolumn{7}{|c|}{} \\
\hline \multicolumn{7}{|c|}{ rand. measurement error percentiles in $\mu \mathrm{m}$} \\
\hline P01 & P05 & P25 & P50 & P75 & P95 & P99 \\
\hline 1 & 1 & 2 & 3 & 3 & 5 & 8 \\
\hline \multicolumn{7}{|c|}{} \\
\hline sys. measurement error percentiles in $\mu \mathrm{m}$ \\
\hline P01 & P05 & P25 & P50 & P75 & P95 & P99 \\
\hline -80 & -60 & -26 & -11 & -2 & 3 & 6 \\
\hline \multicolumn{7}{|c|}{} \\
\hline meas. uncertainty (k=2) & percentiles in $\mu \mathrm{m}$ \\
\hline P01 & P05 & P25 & P50 & P75 & P95 & P99 \\
\hline 3 & 4 & 8 & 24 & 53 & 121 & 163 \\
\hline
\end{tabular}

Figure 8 shows the expanded uncertainty $(k=2)$ for the evaluated measurement series of gear wheel $I D w$. It is observable, that the measurement uncertainty is higher (P50 nearly twice as large as for gear wheel $\left.I D_{\mathrm{DG}}\right)$. The gear roots are also characterized by a very high measurement uncertainty.

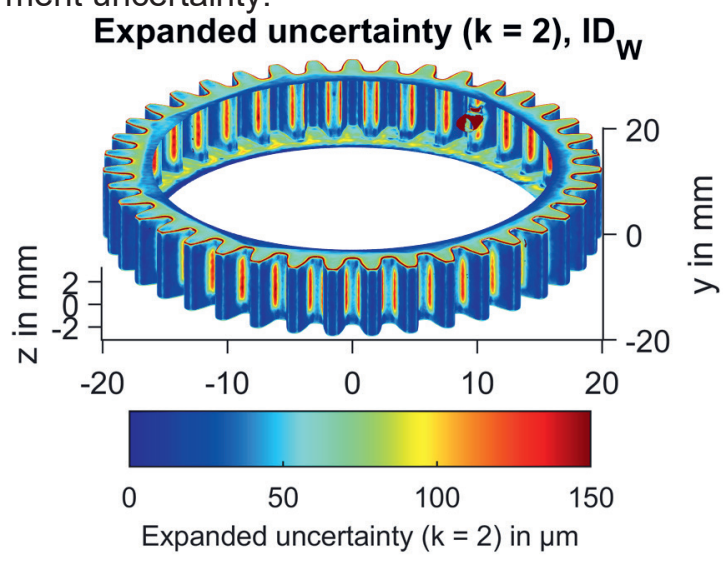

Fig. 8: Visualization of the expanded measurement uncertainty $(k=2)$ of gear wheel IDW

Table 3 confirms that the expanded measurement uncertainty is higher for this coloration setting compared to gear wheel $I D_{\mathrm{DG}}$, although the random part of the measurement error is even lower with P50 only reaching $2 \mu \mathrm{m}$. The systematic work piece deviations of both gear wheels are nearly identical with only minor differences.
Table 3: Statistical view of the calculated work piece deviations and measurement uncertainty contributions (IDw)

\begin{tabular}{|c|c|c|c|c|c|c|}
\hline \multicolumn{7}{|c|}{ sys. work piece deviations percentiles in $\mu \mathrm{m}$} \\
\hline P01 & P05 & P25 & P50 & P75 & P95 & P99 \\
\hline-211 & -199 & -74 & -26 & -4 & 22 & 41 \\
\hline \multicolumn{7}{|c|}{} \\
\hline \multicolumn{7}{|c|}{ rand. measurement error percentiles in $\mu \mathrm{m}$} \\
\hline P01 & P05 & P25 & P50 & P75 & P95 & P99 \\
\hline 1 & 1 & 1 & 2 & 2 & 4 & 6 \\
\hline \multicolumn{7}{|c|}{} \\
\hline sys. measurement error percentiles in $\mu \mathrm{m}$ \\
\hline P01 & P05 & P25 & P50 & P75 & P95 & P99 \\
\hline-97 & -62 & -35 & -22 & -12 & -6 & -3 \\
\hline \multicolumn{7}{|c|}{} \\
\hline meas. uncertainty (k=2) percentiles in $\mu \mathrm{m}$ \\
\hline P01 & P05 & P25 & P50 & P75 & P95 & P99 \\
\hline 8 & 12 & 24 & 44 & 71 & 124 & 201 \\
\hline
\end{tabular}

\section{Discussion}

It could be shown that the measurement of POM gear wheels can in principle be realized by coloration during the manufacturing process. This was demonstrated at hand of two prototypes with different colorizations. Nonetheless, it was also observed that the measurement uncertainty varies on the measured surface, which is possibly influenced by the different inclination angles during the measurements. Especially the limited optical accessibility of the tooth roots presumably lead to increased uncertainty values. This shows that a global illumination setting during the measurement is not optimal for all geometrical features of the gear wheel. Although a complete measurement was possible, the achieved expanded measurement uncertainties of $24 \mu \mathrm{m}$ (P50 of ID $\mathrm{DG}$ ) and $44 \mu \mathrm{m}$ (P50 of $\left.I D_{\mathrm{W}}\right)$ might be insufficient nonetheless. Depending on the defined tolerances for different geometrical features on the polymer gear wheels surface, the "Golden Rule" of metrology might prevent the geometrical inspection of those gear wheels using structured-light scanners. The measurement uncertainty could still be reduced for the presented measurement system by increasing the number of sensor positions and introducing additional illumination settings but the measurement expense would then rise quite substantially. It is worth noting that the calculated precision of the measurements was very good compared to the systematic measurement error. This could be caused by extensive averaging of measurement data due to the high number of recorded projections. Lastly it is noted, that the used reference measurement setup (CT) also exhibits some kind of measurement uncertainty, which was not taken into account within this contribution. A more ac- 
curate determination of the single point measurement uncertainty of structured-light scanners using CT as a reference measurement system must also take this error source into account. The presented work demonstrated the possibility for a better understanding of a certain measurement setup regarding the achieved measurement uncertainty. It is not uncommon in industrial applications that a single measurement setup is operated to monitor the output of a production line. The single point uncertainty provides a flexible tool to verify the measurement results of different measurement setups. Further research efforts could be invested to determine improved coloration options in order to find optimally coordinated optically imaging mechanisms between the gear wheel surface and the projected light of the structured-light scanner.

\section{Acknowledgments}

The authors would like to thank the German Research Foundation (DFG) for supporting the research project "FOR 2271 process-oriented tolerance management based on virtual computer-aided engineering tools" under grant numbers HA 5915/9-1 and DR 421/21-1 and for the financial support of the acquisition of the CT system "Zeiss Metrotom 1500" through Grant No. 324672600.

\section{References}

[1] W. Knapp, "Tolerance and uncertainty," in Laser Metrology and Machine Performance V, 2001, pp. 357-366.

[2] M. Smouni, CZ 3D Metrology Solutions: The Golden Rule - Made by ZEISS. [Online] Available: https://www.cz-3d-metrology.com/2017/12/14/the-golden-rule-made-byzeiss/. Accessed on: Apr. 172019.

[3] G. Berndt, E. Hultzsch, and H. Weinhold, "Funktionstoleranz und Meßunsicherheit," Wissenschaftliche Zeitschrift der Technischen Universität Dresden, vol. 17, no. 2, p. 465, 1968.

[4] Computed tomography in dimensional measurement, VDI/VDE 2630 Part 2.1, 2015.

[5] Geometrical product specifications (GPS) -- Coordinate measuring machines (CMM): Technique for determining the uncertainty of measurement, ISO 15530-3:2011, 2018.

[6] International vocabulary of metrology: Basic and general concepts and associated terms (VIM) German-English version ISO/IEC-Guide 99:2007, Corrected version 2012, 4th ed. Berlin: Beuth, 2012.

[7] A. M. Müller and T. Hausotte, "Comparison of different measures for the single point uncertainty in industrial X-ray computed tomography," in 9th Conference on Industrial Computed Tomography, 2019.

[8] A. M. Müller, F. Wohlgemuth, and T. Hausotte, "Simulation-based correction of systematic errors for CT measurements," in 8th Conference on Industrial Computed Tomography, 2018.
[9] A. M. Müller and T. Hausotte, "Utilization of single point uncertainties for geometry element regression analysis in dimensional X-ray computed tomography," in 9th Conference on Industrial Computed Tomography, 2019.

[10] A. M. Müller, T. Oberleiter, K. Willner, and T. Hausotte, "Implementation of parameterized work piece deviations and measurement uncertainties into performant meta-models for an improved tolerance specification," Proceedings of the International Conference on Engineering Design, ICED, 2019.

[11] A. M. Müller, S. Metzner, and T. Hausotte, "Characterization of the single point precision of steel gear wheel measurements using tactile coordinate measurement machines in scanning mode: accepted contribution," in 20. GMA/ITGFachtagung Sensoren und Messsysteme, 2019.

[12] E. Baur, T. Osswald, and N. Rudolph, Plastics handbook: The resource for plastics engineers, 5th ed. Munich: Hanser, 2019.

[13] G. Menges, G. Wübken, and B. Horn, "Einfluß der Verarbeitungsbedingungen auf die Kristallinität und Gefügestruktur teilkristalliner Spritzgußteile," Colloid and Polymer Science, vol. 254, no. 3, pp. 267-278, 1976.

[14] C. Raymond and S. Ronca, "Relation of Structure to Electrical and Optical Properties," in Brydson's Plastics Materials: Eighth Edition, 2016, pp. 103-125.

[15] A. Müller, Coloring of Plastics: Fundamentals Colorants - Preparations. München: Hanser, Carl, 2012.

[16] GOM GmbH, ATOS Core - Optischer 3D-Scanner. [Online] Available: https://www.gom.com/de/messsysteme/atos/atos-core.html. Accessed on: Apr. 172019.

[17] GOM GmbH, ATOS Professional, 2018.

[18] Carl Zeiss Industrielle Messtechnik, 3D Röntgenmessung in der Qualitätssicherung: ZEISS METROTOM. [Online] Available: https://www.zeiss.de/messtechnik/produkte/systeme/computertomographie/metrotom.html. Accessed on: Apr. 172019.

[19] Volume Graphics GmbH, VGSTUDIO MAX, 2019.

[20] M. Hazewinkel, V. I. Bityutskov, R. V. Gamkrelidze, and J. V.'e. Prohorov, Encyclopaedia of mathematics. Dordrecht: Kluwer academic, 1988-1994.

[21] Joint Committee for Guides in Metrology (JCGM/WG 1), Guide to the expression of uncertainty in measurement: JCGM 100:2008 GUM 1995 with minor corrections, 2008. 